\title{
Master/slave: A better tool for Gabor filtering optical coherence tomography imaging instruments
}

Cernat, Ramona; Bradu, Adrian; Israelsen, Niels Møller; Bang, Ole; Rivet, Sylvain; Keane, Pearse A.; Heath, David Garway; Rajendram, Ranjan; Podoleanu, Adrian

\section{Published in:}

Proceedings of SPIE - The International Society for Optical Engineering

Link to article, DOI:

10.1117/12.2286057

Publication date:

2017

Document Version

Publisher's PDF, also known as Version of record

Link back to DTU Orbit

Citation (APA):

Cernat, R., Bradu, A., Israelsen, N. M., Bang, O., Rivet, S., Keane, P. A., Heath, D. G., Rajendram, R., \& Podoleanu, A. (2017). Master/slave: A better tool for Gabor filtering optical coherence tomography imaging instruments. Proceedings of SPIE - The International Society for Optical Engineering, 2017, [104160N]. https://doi.org/10.1117/12.2286057

\section{General rights}

Copyright and moral rights for the publications made accessible in the public portal are retained by the authors and/or other copyright owners and it is a condition of accessing publications that users recognise and abide by the legal requirements associated with these rights.

- Users may download and print one copy of any publication from the public portal for the purpose of private study or research.

- You may not further distribute the material or use it for any profit-making activity or commercial gain

- You may freely distribute the URL identifying the publication in the public portal 


\title{
Master/slave: a better tool for Gabor filtering optical coherence tomography imaging instruments
}

\author{
Ramona Cernat $^{a}$, Adrian Bradu ${ }^{a}$, Niels Moller Israelsen ${ }^{b}$, Ole Bang ${ }^{b}$, Sylvain Rivet $^{c}$, \\ Pearse A. Keane $^{d}$, David-Garway Heath $^{d}$, Ranjan Rajendram ${ }^{d}$ and Adrian Podoleanu ${ }^{a}$ \\ ${ }^{a}$ Applied Optics Group, University of Kent, Canterbury CT2 7NH, Kent, United Kingdom \\ ${ }^{b}$ DTU Fotonik, Dept. Photonics Eng, Technical University of Denmark, DK-2800, Denmark ${ }^{c}$ Université de Bretagne \\ Occidentale, EA 938 Laboratoire de Spectromtrie et Optique Laser, 6 Avenue Le Gorgeu, C.S. 93837, 29238 Brest Cedex \\ 3, France ${ }^{d}$ NIHR Biomedical Research Centre at Moorfields Eye Hospital NHS Foundation Trust and UCL Institute of \\ Ophthalmology, London, EC1V 9EL United Kingdom \\ a.bradu@kent.ac.uk
}

\begin{abstract}
In this report, the benefits that the Master/Slave (MS) implementation of optical coherence tomography (OCT) can bring to a Gabor filtering (GF) imaging instrument are illustrated. The MS allows simultaneous display of three categories of images in one frame: multiple depth en-face OCT images, two B-scan OCT and a confocal like image. The power of MS is illustrated here by showing 3D images of constant transversal resolution from different objects, obtained by merging sub-volumes collected for four different focus positions. By combining the two techniques, GF and MS, a powerful imaging instrument is demonstrated. We show that when more than four focus positions are required, MS can produce fused volumes faster than the conventional FT based procedure.
\end{abstract}

OCIS codes: $110.4500,170.0180$.

\section{Introduction}

OCT is a non-invasive imaging technology providing high-axial resolution. In opposition to other imaging modalities, in OCT, the axial resolution in the image is decoupled from the lateral resolution. The axial resolution is determined by the bandwidth of the optical source employed. On the other hand, the transversal resolution is dependent on the interface optics in the sample arm of the interferometer, mainly on the numerical aperture of the microscope objective employed to focus light on the sample, as in confocal microscopy.

In OCT, as A-scans are produced via FFTs of the channelled spectra, all points along the A-scan are generated under a single focus adjustment. Consequently, low numerical aperture (NA) interface optics is used to enable a confocal profile width or depth of focus (DOF), comparable with the axial range (AR) of the OCT. If good lateral resolution is needed along the axial depth, by increasing the NA of the interface optics, the confocal gate width becomes narrower than the axial range. The problem presented is that the region of highest quality (as determined by contrast and resolution) is limited by the width and position of the DOF interval within the AR. Efforts have been made to extend the DOF whilst maintaining high lateral resolution by using for example axicon lenses to produce a Bessel beam. A solution suitable for high NA interface optics and applicable to spectral (Fourier) domain OCT was reported based on Gabor filtering [1], where acquisition was repeated for several focus positions, $R=A R / D O F$. An OCT cross-sectional image was acquired at $R=5$ different focus positions dictated by a liquid lens. The in-focus regions were extracted and then spliced together to form a single image wherein all regions are in focus. It was determined that 5 repetitions of data acquisition with shifted focus were sufficient as the DOF interval was approximately $1 / 5$ th of the AR.

In FFT based OCT, to perform GF, the brightest parts of A-scans for each focus position are selected while the rest of A-scans are discarded. The selected parts of A-scans are fused into final B-scans or into a final volume of the tissue. In this way, in the final B-scan, or final volume, better transversal resolution along depth is achieved than when using a single focus position. In other words, for each spectral sweep, the FFT delivers more data than retained in the final image or final volume. Here, we demonstrate that the MS technology [2] is a better tool to be used in a GF instrument than the FFT based method. When using MS, the FFT operation is replaced by multiple comparison operations of the signal at the photo-detector output with replicas of the same signal (masks) corresponding to the photo-detected signal obtained when a mirror is used at different axial positions [2]. For each depth position, different masks can be used.

Optical Coherence Imaging Techniques and Imaging in Scattering Media II, edited by Maciej Wojtkowski, Stephen A. Boppart, Wang-Yuhl Oh, Proc. of SPIE-OSA Vol. 10416, 104160N

(C) 2017 SPIE-OSA · CCC code: 1605-7422/17/\$18 · doi: 10.1117/12.2286057 


\section{Materials and Methods}

The OCT imaging instrument used for this study is similar to that depicted in Fig. 1a, and presented in detail in reference [3]. In the sample arm, an achromatic lens $\mathrm{L}_{1}$ (Fig. 1a) is supported by a miniature linear stage (mTS) [4]. An in-house MS software, is used to display 3 categories of images at each display refresh. Apart from the simultaneous display of 9 enface OCT images, the MS procedure allows assembling a confocal image. The number of en-face images, as well as the differential distance between them, is adjustable, which allows the generation of an adjustable thickness confocal image. Two B-scan images are also presented simultaneously with the display of several en-face OCT images and the confocal lookalike image.

A miniature motorized miniature high-precision linear stage mTS, (model UMS-5 Laser Pacific Equipment, Tustin, CA, under R\&D) [4] was used. The mTS is controlled by a stepper motor controller. The lens $\left(\mathrm{L}_{1}\right)$ used in the object arm (Fig. 1a) is an achromat doublet ( $\mathrm{f}=19 \mathrm{~mm}$ ), glued on a PolyLacticAcid (PLA) block holder created with a 3D printer. The scanning lens $\left(\mathrm{L}_{2}\right)$ contains 2 achromat doublets for enhanced transversal resolution.
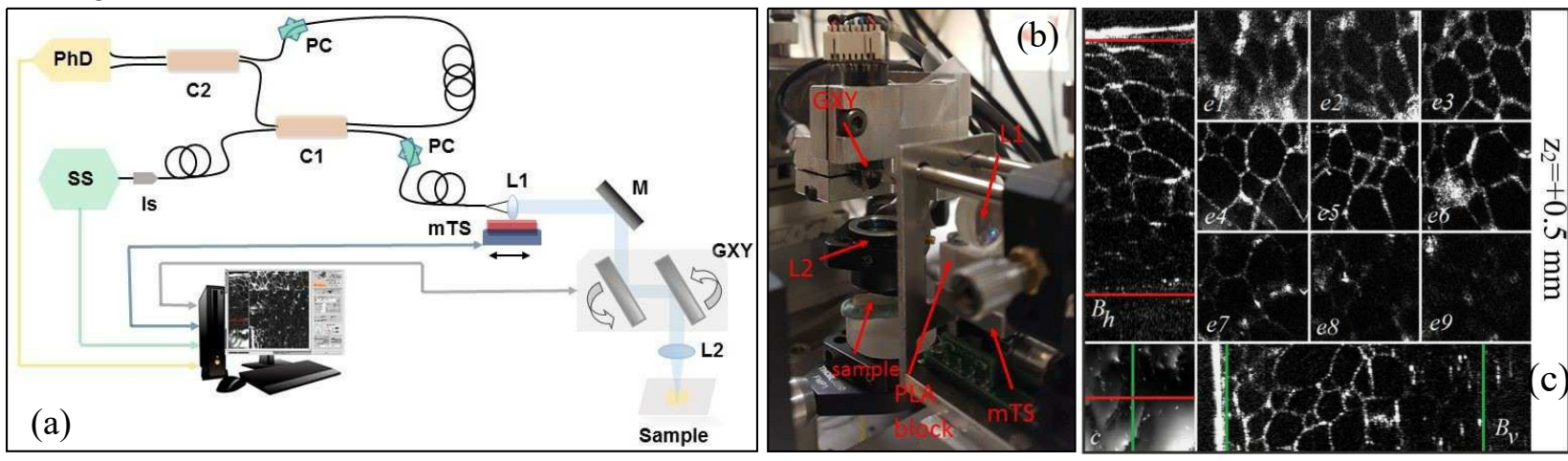

Fig. 1: (a) The miniature linear stage (mTS) used to move lens $\mathrm{L}_{1}$. (b) Photograph of the mTS and its assembly close to the scanning head. (c): MS display for one focus position $\left(\mathrm{z}_{2}\right)$ : 9 en-face OCT images (e1-e9) collected from a piece of cucumber, compound image (c) and $2 \mathrm{~B}$-scans $\left(\mathrm{B}_{h}\right.$ and $\left.\mathrm{B}_{v}\right)$.

\section{Results and Discussion}

The mTS is used to position the focus inside the sample at different axial positions. For each such position, $\mathrm{Q}=200$ en-face OCT images are produced. The Q images can be used to produce volumes. In comparison with conventional FFT based OCT technology, where volumes are assembled from many A-scans, here volumes are assembled from en-face OCT images. As the FFT based technology delivers the whole A-scan for each FFT step, for each focus adjustment, the brightest parts of such A-scans are selected from each A-scans while the rests are discarded. The selected parts of A-scans are then stitched together to form a final volume. In MS, there is no need to cut and stitch A-scans. Simply, because there is a processor for each depth, we only calculate results for the depths within the confocal gate interval (DOF). Only the en-face images of largest brightness are worked out for each focus position. Then these en-face images are assembled into the final volume.

The MS display obtained when imaging a cucumber piece for a single focus position $\left(\mathrm{z}_{2}=0.5 \mathrm{~mm}\right)$ is presented in Fig. $1 \mathrm{c}$ : 9 en-face OCT images, separated axially by $170 \mu \mathrm{m}$, the compound image (left bottom, label c) as well as two orthogonal B-scan OCT images $\left(\mathrm{B}_{h}\right.$ and $\left.\mathrm{B}_{v}\right)$. The size of the en-face images is $0.56 \times 0.56 \mathrm{~mm}^{2}$, while the size of the B-scans is $0.56 \mathrm{~mm}$ (lateral) $\times 2 \mathrm{~mm}$ (depth). The measurements were repeated for 3 extra focus positions. For each position, 3D sub-volumes were produced (Fig. 2). For each sub-volume, a number of 200 axial points were used.

The GF method is extremely time consuming as 3D images must be created for each focusing position before merging the volumes created. If the FFT based OCT method is used, for each position of the focusing beam, large volumes in terms of their axial range need to be created where each A-scan is the result of a succession of sequential mathematical operations (apodization, dispersion compensation algorithms) and finally of the FFT. As only data collected from within the confocal gate is useful for the final image, the FFT based method produces a large amount of discarded data. The MS technique, resolves this inconvenience, as only data from within the confocal gate is processed, hence smaller sub-volumes to be fused at the end of the process of constructing the entire image.

To evaluate the performance of the MS when used with the GF method, we compared the time to produce a fused 3D image using the MS with the time required to produce the same image via FFT, when data was not re-sampled or 

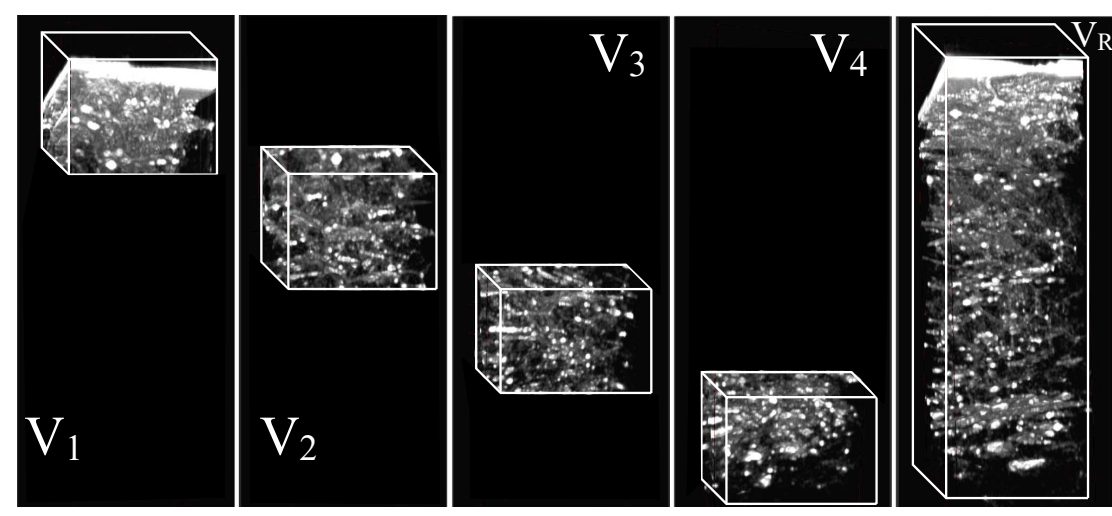

Fig. 2: MS display for 1 focus position of lens L. The MS display consists in 9 en-face OCT images (e1-e9) collected from a piece of cucumber, compound image (c) and two B-scans (Bh and Bv).

compensated for unbalanced dispersion. The time to produce a sub-volume using FFTs is the same irrespective of the number of axial pixels in the sub-volume, so the time to produce the final 3D volume using the FFT method is proportional to the number of sub-volumes. When using the MS method, the smaller the number $Q$ of axial points, less is the computation time. Our benchmarking showed that by using a number of sub-volumes R $>4$, with MS, the process of producing Gabor fused volumes is shorter than using the FFTs.

The use of the MS method allows evaluation of lateral resolution and of sensitivity variation with depth simultaneously, by providing data from multiple-depth en-face images in real time. The combination of GF with the MS method presents several advantages over its combination with a FFT based one:

1. There is no need for cut and stitch of the axial reflectivity profiles. Only data from within the confocal gate is processed (calculations are not performed for depths outside the confocal gate, so no data is discarded).

2. Using the MS procedure, the en-face OCT images can be produced directly. Via the FFT procedure, the whole 3D volume has to be produced first.

3. As no FFTs are employed, there is no need for re-sampling data, or compensate for unbalanced dispersion, hence a faster way of producing images. Even when no re-sampling of data is needed, the time required by the MS procedure combined with GF is less than that of FFT based OCT combined with GF, when the number of sub-volumes to be produced is at least $\mathrm{R}=4$.

4. As MS is tolerant to dispersion in the interferometer, there is no need to balance the dispersion, so there is no need for any dispersion compensation procedure, simplifying in this way the process of assembling the OCT interferometer.

\section{Acknowledgements}

RC, DH, PK, RR and AP are supported by the NIHR Biomedical Research Centre at Moorfields Eye Hospital NHS Foundation Trust and the UCL Institute of Ophthalmology. AP is also supported by the Marie Curie EID UBAPHODESA FP7PEOPLE-2013-ITN 607627, European Research Council (ERC) (AMEFOCT 680879) and by the Royal Society Wolfson Research Merit Award. NMI, OB and AP acknowledge support from Innovation Fund Denmark through the Shape OCT grant No. 4107-00011A. AB and AP are also supported by the EPSRC grant REBOT - EP/N019229/1. SR acknowledges the MC Intra-European Fellowship for Career Development, No. 625509. RC and AB equally contributed to this paper.

\section{References}

1. J.P. Rolland, P. Meemon,S. Murali, K.P. Thompson K. and Lee, "Gabor-based fusion technique for Optical Coherence Microscopy," Opt. Express 18, 3632-3642 (2010).

2. A. Podoleanu and A. Bradu, "Master slave interferometry for parallel spectral domain interferometry sensing and versatile 3D optical coherence tomography," Opt. Express 21, 19324-19338 (2013).

3. R. Cernat, A. Bradu, N. Israelsen, O. Bang, S. Rivet, P.A. Keane, D.G. Heath, R. Rajendram, and A. Podoleanu, "Gabor fusion master slave optical coherence tomography," Biomed. Opt. Express 8, 813-827 (2017).

4. Pacific Laser Equipment website: http://plequipment.com. 